\title{
Autophagy in plasma cell pathophysiology
}

\author{
Laura Oliva ${ }^{1}$ and Simone Cenci ${ }^{1,2,3}$ * \\ 1 Division of Genetics and Cell Biology, San Raffaele Scientific Institute, Milan, Italy \\ 2 Università Vita-Salute San Raffaele, Milan, Italy \\ ${ }^{3}$ Bone Pathophysiology Program (BoNetwork), Division of Genetics and Cell Biology, San Raffaele Scientific Institute, Milan, Italy
}

\section{Edited by:}

Catherine Pellat-Deceunynck, Centre National de la Recherche Scientifique, France

\section{Reviewed by:}

Aaron James Marshall, University of Manitoba, Canada

Laurence Lamy, National Cancer Institute, USA

*Correspondence:

Simone Cenci, San Raffaele Scientific Institute, Via Olgettina 58, Milan

20132, Italy

e-mail:cenci.simone@hsr.it

Plasma cells (PCs) are the effectors responsible for antibody (Ab)-mediated immunity. They differentiate from B lymphocytes through a complete remodeling of their original structure and function. Stress is a constitutive element of PC differentiation. Macroautophagy, conventionally referred to as autophagy, is a conserved lysosomal recycling strategy that integrates cellular metabolism and enables adaptation to stress. In metazoa, autophagy plays diverse roles in cell differentiation. Recently, a number of autophagic functions have been recognized in innate and adaptive immunity, including clearance of intracellular pathogens, inflammasome regulation, lymphocyte ontogenesis, and antigen presentation. We identified a previously unrecognized role played by autophagy in PC differentiation and activity. Following B cell activation, autophagy moderates the expression of the transcriptional repressor Blimp-1 and immunoglobulins through a selective negative control exerted on the size of the endoplasmic reticulum and its stress signaling response, including the essential PC transcription factor, XBP-1. This containment of PC differentiation and function, i.e., Ab production, is essential to optimize energy metabolism and viability. As a result, autophagy sustains Ab responses in vivo. Moreover, autophagy is an essential intrinsic determinant of long-lived PCs in their as yet poorly understood bone marrow niche. In this essay, we discuss these findings in the context of the established biological functions of autophagy, and their manifold implications for adaptive immunity and PC diseases, in primis multiple myeloma.

Keywords: antibody, autophagy, endoplasmic reticulum, multiple myeloma, plasma cell, proteostasis, unfolded protein response, XBP-1

\section{INTRODUCTION}

The biology of plasma cell (PC) differentiation is a unique model for scientists to investigate the complex connections between metabolism, stress, proteome plasticity, and cellular renovation. In particular, the regulation of antibody $(\mathrm{Ab})$ production is a valuable paradigm for the molecular wirings controlling protein folding and assembly in professional secretory cells. Moreover, the bone marrow $\mathrm{PC}$ niche, which provides lifelong Ab memory, depends on as yet incompletely understood intrinsic and environmental components, whose derangement is instrumental to the development of multiple myeloma.

We recently discovered an unanticipated essential function played by autophagy during PC differentiation, disclosing new links with endoplasmic reticulum (ER) homeostasis and $\mathrm{Ab}$ production. Moreover, autophagy emerges as an intrinsic requirement of long-lived PCs and long-term immunity (1). Scope of the present review is to discuss the newly identified role of autophagy in PC pathophysiology, in perspective of recently established autophagic functions across stress biology, cell differentiation, immunity, and cancer.

Abbreviations: $\mathrm{Ab}$, antibody; $\mathrm{Ag}$, antigen; $\mathrm{EM}$, electron microscopy; ER, endoplasmic reticulum; ERAD, ER associated degradation; Ig, immunoglobulin; PC, plasma cell; SILAC, stable isotope labeling in cell culture; SLR, SQSTM1-like receptor; TLR, Toll-like receptor; UPR, unfolded protein response

\section{THE STRESS OF PLASMA CELL DIFFERENTIATION}

Plasma cells are the terminal effectors of adaptive immunity endowed with the unique ability to secrete Abs capable of neutralizing pathogens and toxins. Upon encounter with antigens (Ags), B cells differentiate into short-lived PCs in secondary lymphoid organs (e.g., spleen and lymph nodes). Most of these effector cells die within few days. In addition, T cell-dependent responses induce the germinal center reaction, which generates a second wave of plasmablasts, secreting high-affinity, class-switched Abs, and capable of acquiring lifelong survival in dedicated bone marrow niches. Long-lived PCs maintain immunological memory of Ab-inducing Ags, yielding prompt protection against pathogens and their toxic products (2).

From a biological standpoint, PCs are professional secretory cells dedicated for massive synthesis, assembly, and secretion of Abs. To accomplish this mission, upon activation, B cells must reshape their proteome. To this aim, a powerful genetic program silences B cell identity, through the repression of genes encoding the transcription factors Pax5 and Bcl-6, and establishes PC function, inducing the transcriptional regulators IRF4 and PRDM1/Blimp-1 (3). Early during differentiation, XBP-1, a key ER stress transducer and transcription factor of the unfolded protein response (UPR), drives ER expansion to augment the folding capacity of this organelle and accommodate intensive immunoglobulin $(\mathrm{Ig})$ synthesis in the secretory pathway $(4,5)$. 
In addition to ER stress, other stresses are constitutive of full gear Ab production in PCs. For example, oxidative protein folding causes redox stress, counterbalanced by antioxidant responses (6, 7). After the blastic phase, in post-mitotic PCs, additional stress may ensue from the impossibility of diluting damaged organelles through cell division, as demonstrated in other non-dividing terminally differentiated cells (8). We found that PCs also experience profound proteasome stress (9). Indeed, although Ig-synthetic activity requires intense proteasome-dependent degradation of $\mathrm{Ab}$ byproducts, during their differentiation short-lived PCs display a progressive, remarkable reduction of proteasome expression, which leads to accumulation of poly-ubiquitinated proteins, at the expense of free ubiquitin - an additional stress referred to as ubiquitin stress (10) - and stabilization of proapoptotic factors (11, 12). This apparently paradoxical lack of adaptation may serve as a built-in mechanism to reduce the apoptotic threshold and limit PC lifespan and the duration of Ab responses (13). We also noted that PC differentiation confers exquisite sensitivity to proteasome inhibition, rendering PCs as sensitive to proteasome inhibitors as multiple myeloma cells, disclosing a general characteristic of PCs, rather than a feature of malignancy (9). Attenuating general protein synthesis by the otherwise toxic agent cycloheximide reduces proteasome sensitivity in differentiating plasmablasts, indicating protein synthesis as a key determinant of the proteolytic burden on proteasomes in PCs (14). Such a challenged protein homeostasis (proteostasis) may explain why the first-in-class proteasome inhibitor bortezomib reduced $\mathrm{Ab}$ responses (12) and attenuated autoAb-mediated pathology in a mouse model of lupus (15). Clearly, basic stress biology in PCs is instructive on putative targets against PC dyscrasias (see below).

\section{AUTOPHAGY: FROM BULK DEGRADATION TO SELECTIVE RECYCLING}

Autophagy is a highly conserved self-digestive strategy that envelops cytoplasmic contents in a double-membrane vesicle, the autophagosome, delivered to the lysosome (in animal cells) or to the vacuole (in plant and yeast cells) for subsequent degradation and recycling. The prime function of autophagy in unicellular organisms is to sustain cellular metabolism in conditions of nutritional starvation $(16,17)$. This metabolic role is conserved in metazoa, where autophagy is an essential source of energetic equivalents (18). An exemplar case, autophagy-incompetent newborn mice fail to resist the physiologic early neonatal starvation (19). Autophagy also provides building blocks for cellular renovation, and is crucially involved in differentiation and development $(18,20)$. In mammals, autophagy is essential for embryogenesis (21) and lineage differentiation, as demonstrated, for example, in adipocytes, erythrocytes, and lymphocytes (20).

By contrast with the ubiquitin-proteasome system, autophagy has long been viewed as a bulk non-selective process, with the only exception of chaperone-mediated autophagy. Its recently recognized capacity to ensure cellular quality control by clearing toxic and damaged macromolecules and organelles disclosed an unanticipated level of selectivity $(22,23)$. Selective autophagic degradation has been reported for a number of endogenous supramolecular structures: peroxisomes (pexophagy) (24), protein aggregates (aggrephagy) (25-27), ribosomes (ribophagy) (28), mitochondria (mitophagy) (29-31), lipid droplets (lipophagy) (32), secretory granules (zymophagy) (33), and midbody remnants after cytodieresis (34).

To target selected cargoes, autophagy makes use of adapter proteins acting as receptors. To mediate selective autophagy, these proteins must: (i) recognize substrates via a ubiquitin-binding activity; (ii) cross-link the cargo with the autophagic machinery via an LC3-interacting region; and (iii) polymerize $(23,35)$. Ubiquitination is thus used not only to convey individual proteins to the proteasome, but also for selective recognition by autophagic receptors, e.g., p62 and NBR1 during mitophagy and aggrephagy. While the prime tag for proteasomal degradation is a chain of ubiquitins covalently linked through their K48 lysine residues, K63linked poly-ubiquitin tags may be preferentially associated with autophagic degradation, although additional post-translational modifications may contribute to direct cargoes to autophagy (23). In most cases, the ubiquitin ligases involved in autophagy remain to be identified. Hitherto established mammalian autophagic receptors include SQSTM1/p62, NBR1, optineurin, NDP52, and Nix. Adaptor proteins are also being characterized, which interact with autophagy receptors to recruit and assemble more Atg proteins, so as to shape the growing autophagosome (phagophore) around the cargo (36).

Virtually, all cellular membranes have been proposed to contribute to autophagosome biogenesis. Among them, the ER is an established membrane source for the phagophore (37). The ER may also undergo autophagic degradation: autophagic trimming of excess ER (reticulophagy, ER-phagy) counterbalances pharmacological stress-induced ER expansion in yeast (38). More recently, a mechanism mediating both mitophagy and ER-phagy has been described in HeLa cells (39). However, defining the physiological significance of reticulophagy in mammals is biologically relevant. As described below, our recent work on autophagy in PCs furthers this view by defining ER-phagy as an essential determinant of PC biology and $\mathrm{Ab}$ immunity (1).

\section{ROLES OF AUTOPHAGY IN INNATE AND ADAPTIVE IMMUNITY}

In the immune system, autophagy serves diverse innate and adaptive functions, including microbe clearance, Ag presentation, and the regulation of inflammation and lymphocyte development (40-42). The co-optation of autophagy to destroy intracellular microbes, i.e., xenophagy, already present in unicellular organisms (43), likely represents the most ancient form of immune defense. Xenophagy has been shown to restrict the growth of bacteria (L. monocytogenes, S. flexnerii, S. typhimurium) (42). The infectious phagosome is intracellularly recognized through internal toll-like receptor (TLR) signaling (44). Then, infected cells can promote phagosome-lysosome fusion or target cytosol-invading bacteria for autophagic degradation (36). A number of autophagic receptors, including SQSTM1/p62, NDP52, and optineurin, have been shown to specifically recognize ubiquitinated bacteria within the cytosol $(23,36)$, hence the idea that SQSTM1-like receptors (SLRs) constitute a new family of innate pattern recognizing receptors (45). Autophagy may play additional antimicrobial activities through SLRs, e.g., by generating microbicidal peptides via incomplete digestion of ribosomal protein precursors 
during M. tuberculosis infection (46). Autophagy also mediates viral recognition and destruction. For example, capsid proteins of the neurotropic Sindbis virus are degraded via p62-dependent autophagy (47).

Autophagy is also involved in the modulation of the inflammatory response. In particular, autophagy may both stimulate and inhibit the activity and output of the inflammasome. While basal autophagy prevents inflammation, e.g., by limiting mitochondrial generation of reactive oxygen species and the resulting inflammasome activation $(48,49)$, induction of autophagy can promote inflammation, mediating the inflammasome-dependent unconventional release of the endogenous pyrogen, IL-1 $\beta$, which in turn can intensify autophagy (50). Autophagy may also yield negative feedback loops to prevent destructive inflammation, e.g., moderating IL- $1 \beta$ release by targeting inflammasomes and pro-IL- $1 \beta$ for degradation $(51,52)$.

Autophagy also serves adaptive immune functions, including the regulation of lymphocyte ontogenesis and homeostasis. Atg proteins have been shown to maintain normal numbers of $\mathrm{CD}^{+}$ and $\mathrm{CD}^{+} \mathrm{T}$ cells, and fetal hematopoietic stem cells (41, 53, 54). First, Atg $5^{-1-}$ bone marrow chimeric mice revealed defects in $\mathrm{T}$ cell development and peripheral homeostasis, and impaired activation-induced proliferation (55). Although activated T cells require autophagy, negative controls may come into play to temper autophagy, preventing detrimental effects. Indeed, components of the extrinsic apoptotic cascade, namely FADD and caspase 8, were found to limit autophagy by interacting with the Atg5-Atg12 complex, thereby sustaining viability of activated T cells (56). In following studies, the development of mature $\mathrm{T}$ cells was found to require an active negative control on the intracellular production of reactive oxygen species, which in turn relies on efficient mitophagy $(55,57)$. Such mitochondrial quality control maintains mature naïve $\mathrm{T}$ cell homeostasis through Beclin-1 stabilization by the class III phosphoinositide-3 kinase Vps34 $(58,59)$. Moreover, in activated T cells, autophagy is induced to maintain ATP levels, proliferation, and the release of cytokines (60).

Autophagy is also important for B cell development: irradiated Rag $1^{-/-}$recipients repopulated with fetal liver progenitors lacking the essential autophagic factor Atg 5 have low counts of peritoneal B-1 B cells, due to defective transition of pro- to pre-B cells (61). Moreover, mice with conditional deletion of Atg5 in mature B cells (Atg $5 / f$ CD19-Cre) show normal numbers of mature B lymphocytes and a normal ratio of marginal zone to follicular B cells, but reduced maintenance of B-1a cells in the periphery $(1,61)$.

A number of studies have implicated autophagy in different Ag presentation pathways. The delivery of exogenous Ags for MHC class II presentation to $\mathrm{CD}^{+} \mathrm{T}$ cells has been shown to depend on autophagy (62). Indeed, MHC class II-loading compartments receive continuous input from autophagosomes, and autophagy has been shown to positively control $\mathrm{CD}^{+}{ }^{+} \mathrm{T}$ cell priming (63-65). Moreover, thymic epithelial cells deliver self Ags to MHC class II-loading compartments through the autophagic machinery. This task is essential to build self-tolerance, as its disruption leads to defective elimination of autoreactive $\mathrm{T}$ cells and autoimmunity (66). Furthermore, autophagy has been shown to mediate $\mathrm{CD}^{+} \mathrm{T}$ cell priming in vivo through cross-presentation of phagocytosed Ags, normally routed through the MHC class II pathway, on MHC class I $(67,68)$. However, autophagy is not a universal Ag-presenting pathway, as we proved it dispensable for presentation by $\mathrm{B}$ cells to cognate $\mathrm{T}$ cells in the germinal center (see below) (1).

We hypothesized that autophagy may play an additional adaptive immune function in terminal PC differentiation, based on the specific biology of Ab-secreting cells (9). First, PC differentiation is expected to require a high degree of proteome plasticity. In support of this notion, we had generated quantitative evidence that both protein translation and degradation increase remarkably in primary activated B cells (14). Second, we had observed that such an increased demand for protein degradation is not met by a corresponding increase in proteasome capacity, which instead decreases dramatically $(11,12,14)$, and reasoned that this would call for complementary protein degradation routes. Having the capacity to compensate for proteasome insufficiency (69), autophagy was an obvious candidate. Third, most, if not all, stresses experienced by PCs are known to be relieved by autophagy $(9,22)$. The following paragraphs illustrate our findings, unveiling the crucial role served by autophagy in the differentiation, function, and viability of PCs, required for humoral immunity, and the underlying mechanism, linking ER homeostasis with Ig synthesis and energy metabolism.

\section{AUTOPHAGY SUSTAINS Ab RESPONSES AND IS ESSENTIAL TO LONG-LIVED PCs}

When we assessed overall autophagic activity in differentiating PCs, we found strong induction of autophagy following B cell activation, both ex vivo and in vivo. In keeping with a developmental program, similar to UPR transcripts, Atg mRNAs increased concertedly during PC differentiation. The use of GFP-LC3 transgenic mice revealed intense autophagy also in long-lived bone marrow PCs (1). Encouraged by these observations, to assess the functional relevance of autophagy in PC ontogenesis, we first investigated $\mathrm{Ab}$ responses in $A \operatorname{tg} 5^{/ / f} \mathrm{CD} 19$-Cre mice. These mice showed reduced IgM and IgG responses in both T-independent and T-dependent immunization experiments, demonstrating a positive role of autophagy in $\mathrm{Ab}$ responses mediated by shortlived PCs. A parallel independent study confirmed these findings, by showing significantly diminished Ab titers in the same mouse model during Ag-specific immunization, parasitic infection, and mucosal inflammation (70).

Inspired by the observation of high autophagic activity also in long-lived PCs, we then asked whether Atg5 is required for longterm humoral immunity, by assessing if Atg $5^{f / f} \mathrm{CD} 19$-Cre mice show defects in bone marrow PC populations. These mice had normal bone marrow PC counts, apparently arguing against a role for autophagy in long-lived PCs. However, the genomic quantification of Cre-mediated deletion of Atg5 disclosed that while in splenic B cells most Atg5 alleles had undergone Cre-dependent recombination, bone marrow PCs displayed normal amounts of the non-deleted allele. Hence, an efficient Darwinian selection for autophagy-competent PCs had occurred, demonstrating that autophagy is absolutely required to establish or maintain longlived PCs. Moreover, despite a normal size of the bone marrow PC pool, Atg $5^{f / f} \mathrm{CD} 19$-Cre mice revealed a defect in long-term Ab immunity, as they had virtually absent Ag-specific long-lived PCs 
in the bone marrow 11 months after T-dependent immunization. Altogether, the data establish autophagy as a novel determinant of the PC memory compartment (1).

Being essential to generate class-switched and high-affinity memory B cells and long-lived PCs, we also checked the germinal center reaction in NP-CGG-immunized Atg $5^{f / f} \mathrm{CD} 19$-Cre mice, but found it normal (1). This evidence mapped the requirement of autophagy specifically to PCs. Moreover, since the germinal center response requires $\mathrm{Ag}$ presentation by $\mathrm{B}$ cells to cognate $\mathrm{T}$ cells, this data demonstrated that autophagy is dispensable for soluble Ag presentation in B cells, in spite of its Ag-presenting role in other contexts (discussed above).

The above findings further our understanding of the intrinsic molecular competence required for PCs to achieve extended survival in the bone marrow (2). Hitherto recognized components of such competence comprise the chromatin modifier Aiolos (71), the transcriptional regulators Blimp-1 (72) and XBP-1 (73), and the anti-apoptotic molecule Mcl-1 (74). The identification of autophagy as a novel molecular requirement of bone marrow PCs and of long-lived humoral immunity is in keeping with its established capacity to grant extended survival to quiescent progenitors and highly specialized terminally differentiated cell types, such as neurons (75).

An important matter of future investigation is the precise level at which autophagy is required in memory $\mathrm{PC}$ ontogenesis, i.e., the survival of non-resident long-lived plasmablasts, their migration to the bone marrow, or the maintenance of resident long-lived PCs in the medullary niche. Moreover, it would be interesting to determine if autophagy also plays a role in maintaining the other memory compartment of B cell immunity, i.e., non-Ig-secreting memory B cells.

\section{AUTOPHAGY CONTAINS PC DIFFERENTIATION AND Ab PRODUCTION THROUGH SELECTIVE ER-PHAGY}

The easiest conceivable explanation for autophagy sustaining $\mathrm{Ab}$ immunity was to hypothesize it to be required for PC differentiation. Confuting this hypothesis, Atg5-deficient B cells apparently underwent normal PC differentiation (1). The exact molecular role of autophagy in developing PCs was gaged by an unbiased comparison of the proteome of autophagy-competent vs. incompetent activated B cells by stable isotope labeling in cell culture (SILAC). Importantly, the proteome of differentiating PCs was completely labeled in as little as 3 days upon activation, not only enabling this approach, but also convincingly demonstrating the highest proteome plasticity inherent to this differentiation program. SILAC proteomics of Atg $5^{-1-}$ PCs revealed a selective and rather exclusive expansion of the ER proteome, including Igs. An autophagic regulation of the size of the ER was demonstrated by two independent electron microscopy (EM) approaches: classical EM and an EM cytochemistry technique designed to stain and unbiasedly quantify the ER (76). Short treatment with distal autophagy inhibitors was sufficient to increase ER proteins in wild type differentiating PCs, unveiling the first case of physiologic reticulophagy in mammals (1).

Attesting to the functional relevance of the identified autophagic regulation of the ER in PC differentiation, Atg5 $5^{-1-}$ PCs had higher UPR signaling than wild type PCs, associated

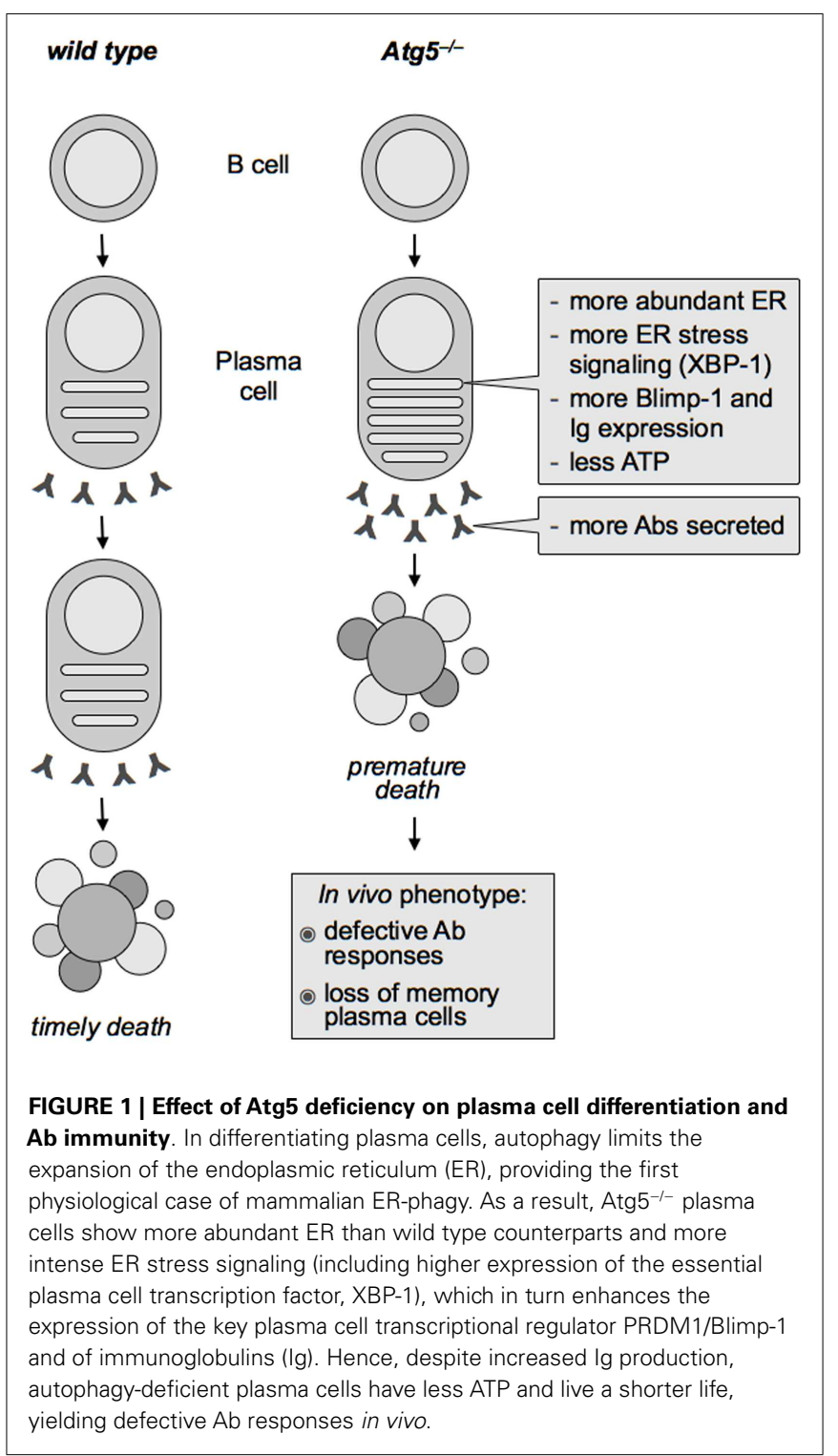

with higher expression of Blimp-1 and Ig transcripts, indicating that autophagy restricts the expression of two key determinants of PC differentiation, XBP-1 and Blimp-1, and of Igs. Providing mechanistic insight, pharmacological ER stress in wild type differentiating PCs was sufficient to increase Blimp-1 and Ig expression beyond the putatively maximal levels associated to PC differentiation. As a result, Atg5 $5^{-1-}$ PCs translated, assembled, and secreted more Abs over time, disclosing an unsuspected regulatory circuit of PC function negatively controlled by autophagy $(1,75)$ (see Figure 1). It will be important to dissect the mechanisms underlying ER-phagy during PC differentiation.

In cellular models of protein folding diseases, autophagy has been shown to dispose of polymeric misfolded protein aggregates in the ER, constituting an alternative form of ER associated degradation (ERAD) $(77,78)$. Noticeably, instead, in differentiating PCs, autophagy does not serve a similar quality control function in the secretory pathway, nor does it remove dysfunctional ER, as 
Atg5 $5^{-1-}$ PCs show normal Ig assembly, and do not accumulate Ig aggregates, but rather display higher capacity in their expanded secretory apparatus (1).

These findings imply that PCs are programed to become more productive $\mathrm{Ab}$ factories than actually observed, with autophagy acting as a physiologic brake on their differentiation and function. How is this reconciled with the defective $\mathrm{Ab}$ responses mounted by $\operatorname{Atg} 5^{f / f} \mathrm{CD} 19-C r e$ mice? This paradox is solved by the observation that Atg5 $5^{-1-}$ PCs have less ATP and live a shorter life than wild type counterparts. Hence, autophagy accomplishes a sensible trade-off between viability and function, setting Ab production to sustainable levels $(1,75)$.

Can the higher Ig-secreting potential of PCs be demonstrated in vivo? A proof-of-principle experiment was the immunization with the T-independent hapten NP-ficoll, which, in our hands, yielded higher anti-NP Ig titers in $A \operatorname{tg} 5 / / f \mathrm{CD} 19-\mathrm{Cre}$ mice, despite normal PC counts, in line with the higher secretory activity of Atg5 ${ }^{-1-}$ PCs observed ex vivo. Unlike other Ags, NP-ficoll has been shown to persist and cause continual B cell activation (79). Hence, repeated rounds of PC differentiation may have surpassed the otherwise dominant impact of reduced $\mathrm{PC}$ viability on $\mathrm{Ab}$ titers, witnessing the hypersecretory effect of Atg5 deficiency at the single PC level $(1,75)$.

The discovery of a novel autophagy-centered regulatory network balancing PC activity and survival in vivo implies an unsuspected plasticity of $\mathrm{Ab}$ responses, potentially exploitable to tune their duration and intensity. A number of immune signaling molecules can regulate autophagy $(41,45)$, supporting this possibility, and offering opportunities to search for molecular targets to modulate $\mathrm{Ab}$ responses, of therapeutic use against autoimmune diseases.

\section{AUTOPHAGY IN MULTIPLE MYELOMA}

A matter of intense scientific debate, the role of autophagy in cancer is complex. Genetic defects of autophagy have been linked with tumorigenesis, establishing the notion that autophagy is a tumor suppressive pathway (80). Oncosuppressive mechanisms of autophagy include protection against the accumulation of oncogenic mutations (81-83) and reactive oxygen species, mainly through mitochondrial homeostasis, and reduction of necrosis and local inflammation (84). While in healthy cells, autophagy may suppress tumor initiation, established cancers may subvert autophagy to cope with intrinsic (e.g., metabolic), environmental (e.g., hypoxic), or pharmacological stress (e.g., induced by cytotoxic agents). This may explain why pharmacological inhibition of autophagy may be beneficial against cancer, being toxic to tumor cells and sensitizing them to chemotherapy $(80,85)$.

Multiple myeloma is a valuable model to investigate the role of autophagy in cancer, especially in perspective of integrated cancer proteostasis. Indeed, myeloma represents the paradigmatic neoplasm responsive to proteasome inhibitors, prototypical negative proteostasis regulators, although a substantial proportion of patients fail to respond, and resistance inevitably ensues (9, 8688). We demonstrated that the exquisite proteasome sensitivity of normal and malignant PCs stems from an unfavorable ratio between proteasome workload and overall capacity $(9,11,12)$, and termed this feature proteostenosis (13). Moreover, we found that myelomas with the highest sensitivity to proteasome inhibition are those expressing fewer active proteasomes in spite of the highest degradative workload, both features being causal to such inherent vulnerability (89). Noticeably, the degradative burden is a relatively neglected source of cellular stress, particularly in cancer (90). In myeloma, we found that recent protein synthesis saturates the limited capacity of the ubiquitin-proteasome system, causing the buildup of ubiquitin conjugates, and is a crucial determinant of proteasome stress (14). It is noteworthy that lymphoplasmocytic lymphoma (Waldenstrom's macroglobulinemia), another B cell cancer known to produce high levels of IgM, also proved vulnerable to proteasome inhibition (91-93), and the clinical use of bortezomib yielded encouraging results $(94,95)$.

Autophagy and the ubiquitin-proteasome system are integrated strategies that cooperate to maintain cellular proteostasis (69). This notion is sufficient to predict that multiple myeloma and Waldenstrom's macroglobulinemia may be as dependent on autophagy as they are on the ubiquitin-proteasome system. This prompted different laboratories to target autophagy in order to overcome resistance to proteasome inhibitors and achieve myeloma cell death, with controversial results. While the blockade of autophagy appears toxic against human myeloma lines, the combined inhibition of the proteasome and autophagy may be synergic or antagonistic, depending on the molecular level of autophagic inhibition (88, 96-98). Experimental discrepancies may be partly explained by the recent discovery of a vital circuit blocking autophagy, disclosing that deregulated autophagy can turn maladaptive in multiple myeloma cells. In brief, human myeloma lines depend for their survival on IRF4, which, through a caspase 10-dependent mechanism, prevents excessive autophagy from executing non-apoptotic myeloma cell death (99). This data suggests that autophagy may play both adaptive and maladaptive roles in myeloma, depending on its intensity, or its targets. A better understanding of the basal, adaptive function of autophagy in myeloma cells is needed to harness this pathway against cancer. Our identification of a new homeostatic function of autophagy, essential for the maintenance of long-lived PCs in the bone marrow, the normal counterpart of multiple myeloma $(1,75)$, prompts to test if PC tumors are at least as dependent on autophagy for their survival, and provides a framework for dissecting the precise function of autophagy in normal and malignant bone marrow PCs. The diverse homeostatic activities of autophagy in different lineages and diseases hitherto defined suggests that PC-specific, and possibly myeloma-specific, autophagic circuits may be identified, linking organelle homeostasis, stress responses, and energy metabolism, to disclose new molecular therapeutic targets.

\section{ACKNOWLEDGMENTS}

We thank Federica Loro and all our lab members for creative discussions. We are particularly grateful to Holger Auner, Paolo Cascio, Stefano Casola, Marco Colonna, Paolo Dellabona, Luigi Maiuri, Giampaolo Merlini, Anna Mondino, Andrea Orsi, Giovanni Palladini, Niccolò Pengo, Fulvio Reggiori, Eelco van Anken, Skip Virgin, and Kathy Weilbaecher. Work in the Cenci Lab was supported by the Multiple Myeloma Research Foundation (Senior Research Award 2010), the Italian Ministry of Health (Giovani Ricercatori 1143560), and the Italian Association for Cancer 
Research (AIRC, Investigator Grant 14691 and Special Program Molecular Clinical Oncology 5 per mille n. 9965).

\section{REFERENCES}

1. Pengo N, Scolari M, Oliva L, Milan E, Mainoldi F, Raimondi A, et al. Plasma cells require autophagy for sustainable immunoglobulin production. Nat Immunol (2013) 14:298-305. doi:10.1038/ni.2524

2. Radbruch A, Muehlinghaus G, Luger EO, Inamine A, Smith KGC, Dorner T, et al. Competence and competition: the challenge of becoming a long-lived plasma cell. Nat Rev Immunol (2006) 6:741-50. doi:10.1038/nri1886

3. Nutt SL, Taubenheim N, Hasbold J, Corcoran LM, Hodgkin PD. The genetic network controlling plasma cell differentiation. Semin Immunol (2011) 23:341-9. doi:10.1016/j.smim.2011.08.010

4. Ron D, Walter P. Signal integration in the endoplasmic reticulum unfolded protein response. Nat Rev Mol Cell Biol (2007) 8:519-29. doi:10.1038/nrm2199

5. Shapiro-Shelef M, Calame K. Regulation of plasma cell development. Nat Rev Immunol (2005) 5:230-42. doi:10.1038/nri1572

6. Garcia-Manteiga JM, Mari S, Godejohann M, Spraul M, Napoli C, Cenci S, et al. Metabolomics of B to plasma cell differentiation. J Proteome Res (2011) 10:4165-76. doi:10.1021/pr200328f

7. Bertolotti M, Sitia R, Rubartelli A. On the redox control of B lymphocyte differentiation and function. Antioxid Redox Signal (2012) 16:1139-49. doi:10.1089/ars.2011.4252

8. Terman A, Kurz T, Navratil M, Arriaga E, Brunk U. Mitochondrial turnover and aging of long-lived postmitotic cells: the mitochondrial-lysosomal axis theory of aging. Antioxid Redox Signal (2010) 12:503-34. doi:10.1089/ars.2009.2598

9. Cenci S. The proteasome in terminal plasma cell differentiation. Semin Hematol (2012) 49:215-22. doi:10.1053/j.seminhematol.2012.04.005

10. Hanna J, Meides A, Zhang DP, Finley D. A ubiquitin stress response induces altered proteasome composition. Cell (2007) 129:747-59. doi:10.1016/j.cell. 2007.03.042

11. Cenci S, Mezghrani A, Cascio P, Bianchi G, Cerruti F, Fra A, et al. Progressively impaired proteasomal capacity during terminal plasma cell differentiation. EMBO J (2006) 25:1104-13. doi:10.1038/sj.emboj.7601009

12. Cascio P, Oliva L, Cerruti F, Mariani E, Pasqualetto E, Cenci S, et al. Dampening $\mathrm{Ab}$ responses using proteasome inhibitors following in vivo B cell activation. Eur J Immunol (2008) 38:658-67. doi:10.1002/eji.200737743

13. Cenci S, van Anken E, Sitia R. Proteostenosis and plasma cell pathophysiology. Curr Opin Cell Biol (2011) 23:216-22. doi:10.1016/j.ceb.2010.11.004

14. Cenci S, Oliva L, Cerruti F, Milan E, Bianchi G, Raule M, et al. Pivotal Advance: protein synthesis modulates responsiveness of differentiating and malignant plasma cells to proteasome inhibitors. J Leukoc Biol (2012) 92:921-31. doi:10.1189/jlb.1011497

15. Neubert K, Meister S, Moser K, Weisel F, Maseda D, Amann K, et al. The proteasome inhibitor bortezomib depletes plasma cells and protects mice with lupuslike disease from nephritis. Nat Med (2008) 14:748-55. doi:10.1038/nm1763

16. Lum JJ, DeBerardinis RJ, Thompson CB. Autophagy in metazoans: cell survival in the land of plenty. Nat Rev Mol Cell Biol (2005) 6:439-48. doi:10.1038/ nrm 1660

17. He C, Klionsky DJ. Regulation mechanisms and signaling pathways of autophagy. Annu Rev Genet (2009) 43:67-93. doi:10.1146/annurev-genet102808-114910

18. Mizushima N, Komatsu M. Autophagy: renovation of cells and tissues. Cell (2011) 147:728-41. doi:10.1016/j.cell.2011.10.026

19. Kuma A, Hatano M, Matsui M, Yamamoto A, Nakaya H, Yoshimori T, et al. The role of autophagy during the early neonatal starvation period. Nature (2004) 432:1032-6. doi:10.1038/nature03029

20. Mizushima N, Levine B. Autophagy in mammalian development and differentiation. Nat Cell Biol (2010) 12:823-30. doi:10.1038/ncb0910-823

21. Tsukamoto S, Kuma A, Murakami M, Kishi C, Yamamoto A, Mizushima N. Autophagy is essential for preimplantation development of mouse embryos. Science (2008) 321:117-20. doi:10.1126/science.1154822

22. Kroemer G, Marino G, Levine B. Autophagy and the integrated stress response. Mol Cell (2010) 40:280-93. doi:10.1016/j.molcel.2010.09.023

23. Johansen T, Lamark T. Selective autophagy mediated by autophagic adapter proteins. Autophagy (2011) 7:279-96. doi:10.4161/auto.7.3.14487

24. Iwata J-I, Ezaki J, Komatsu M, Yokota S, Ueno T, Tanida I, et al. Excess peroxisomes are degraded by autophagic machinery in mammals. J Biol Chem (2006) 281:4035-41. doi:10.1074/jbc.M512283200
25. Szeto J, Kaniuk NA, Canadien V, Nisman R, Mizushima N, Yoshimori T, et al. ALIS are stress-induced protein storage compartments for substrates of the proteasome and autophagy. Autophagy (2006) 2:189-99.

26. Filimonenko M, Isakson P, Finley KD, Anderson M, Jeong H, Melia TJ, et al. The selective macroautophagic degradation of aggregated proteins requires the PI3Pbinding protein Alfy. Mol Cell (2010) 38:265-79. doi:10.1016/j.molcel.2010.04. 007

27. Komatsu M, Waguri S, Koike M, Sou Y-S, Ueno T, Hara T, et al. Homeostatic levels of p62 control cytoplasmic inclusion body formation in autophagy-deficient mice. Cell (2007) 131:1149-63. doi:10.1016/j.cell.2007.10.035

28. Kraft C, Deplazes A, Sohrmann M, Peter M. Mature ribosomes are selectively degraded upon starvation by an autophagy pathway requiring the Ubp3p/Bre5p ubiquitin protease. Nat Cell Biol (2008) 10:602-10. doi:10.1038/ncb1723

29. Okamoto K, Kondo-Okamoto N, Ohsumi Y. Mitochondria-anchored receptor Atg32 mediates degradation of mitochondria via selective autophagy. Dev Cell (2009) 17:87-97. doi:10.1016/j.devcel.2009.06.013

30. Geisler S, Holmstrom KM, Skujat D, Fiesel FC, Rothfuss OC, Kahle PJ, et al. PINK1/Parkin-mediated mitophagy is dependent on VDAC1 and p62/SQSTM1. Nat Cell Biol (2009) 12:119-31. doi:10.1038/ncb2012

31. Novak I, Kirkin V, McEwan DG, Zhang J, Wild P, Rozenknop A, et al. Nix is a selective autophagy receptor for mitochondrial clearance. EMBO Rep (2010) 11:45-51. doi:10.1038/embor.2009.256

32. Singh R, Kaushik S, Wang Y, Xiang Y, Novak I, Komatsu M, et al. Autophagy regulates lipid metabolism. Nature (2009) 458:1131-5. doi:10.1038/ nature07976

33. Grasso D, Ropolo A, Lo Re A, Boggio V, Molejon MI, Iovanna JL, et al. Zymophagy, a novel selective autophagy pathway mediated by VMP1-USP9xp62, prevents pancreatic cell death. J Biol Chem (2011) 286:8308-24. doi:10. 1074/jbc.M110.197301

34. Kuo T-C, Chen C-T, Baron D, Onder TT, Loewer S, Almeida S, et al. Midbody accumulation through evasion of autophagy contributes to cellular reprogramming and tumorigenicity. Nat Cell Biol (2011) 13:1214-23. doi:10.1038/ncb2332

35. Birgisdottir AB, Lamark T, Johansen T. The LIR motif - crucial for selective autophagy. J Cell Sci (2013) 126:3237-47. doi:10.1242/jcs. 126128

36. Reggiori F, Komatsu M, Finley K, Simonsen A. Selective types of autophagy. Int J Cell Biol (2012) 2012:156272. doi:10.1155/2012/156272

37. Hayashi-Nishino M, Fujita N, Noda T, Yamaguchi A, Yoshimori T, Yamamoto A. A subdomain of the endoplasmic reticulum forms a cradle for autophagosome formation. Nat Cell Biol (2009) 11:1433-7. doi:10.1038/ncb1991

38. Bernales S, McDonald KL, Walter P. Autophagy counterbalances endoplasmic reticulum expansion during the unfolded protein response. PLoS Biol (2006) 4:e423. doi:10.1371/journal.pbio.0040423

39. Hanna RA, Quinsay MN, Orogo AM, Giang K, Rikka S, Gustafsson AB. Microtubule-associated protein 1 light chain 3 (LC3) interacts with Bnip3 protein to selectively remove endoplasmic reticulum and mitochondria via autophagy. J Biol Chem (2012) 287:19094-104. doi:10.1074/jbc.M111.322933

40. Saitoh T, Akira S. Regulation of innate immune responses by autophagy-related proteins. J Cell Biol (2010) 189:925-35. doi:10.1083/jcb.201002021

41. Levine B, Mizushima N, Virgin HW. Autophagy in immunity and inflammation. Nature (2011) 469:323-35. doi:10.1038/nature09782

42. Deretic V. Autophagy as an innate immunity paradigm: expanding the scope and repertoire of pattern recognition receptors. Curr Opin Immunol (2012) 24:21-31. doi:10.1016/j.coi.2011.10.006

43. Fujita N, Yoshimori T. Ubiquitination-mediated autophagy against invading bacteria. Curr Opin Cell Biol (2011) 23:492-7. doi:10.1016/j.ceb.2011.03.003

44. Sanjuan MA, Dillon CP, Tait SWG, Moshiach S, Dorsey F, Connell S, et al. Tolllike receptor signalling in macrophages links the autophagy pathway to phagocytosis. Nature (2007) 450:1253-7. doi:10.1038/nature06421

45. Deretic V, Saitoh T, Akira S. Autophagy in infection, inflammation and immunity. Nat Rev Immunol (2013) 13:722-37. doi:10.1038/nri3532

46. Ponpuak M, Davis AS, Roberts EA, Delgado MA, Dinkins C, Zhao Z, et al. Delivery of cytosolic components by autophagic adaptor protein p62 endows autophagosomes with unique antimicrobial properties. Immunity (2010) 32:329-41. doi:10.1016/j.immuni.2010.02.009

47. Sumpter R Jr, Levine B. Selective autophagy and viruses. Autophagy (2011) 7:260-5. doi:10.4161/auto.7.3.14281

48. Zhou R, Yazdi AS, Menu P, Tschopp J. A role for mitochondria in NLRP3 inflammasome activation. Nature (2011) 469:221-5. doi:10.1038/nature09663

49. Nakahira K, Haspel JA, Rathinam VAK, Lee S-J, Dolinay T, Lam HC, et al. Autophagy proteins regulate innate immune responses by inhibiting the release 
of mitochondrial DNA mediated by the NALP3 inflammasome. Nat Immunol (2010) 12:222-30. doi:10.1038/ni.1980

50. Dupont N, Jiang S, Pilli M, Ornatowski W, Bhattacharya D, Deretic V. Autophagy-based unconventional secretory pathway for extracellular delivery of IL-1beta. EMBO J (2011) 30:4701-11. doi:10.1038/emboj.2011.398

51. Harris J, Hartman M, Roche C, Zeng SG, O’Shea A, Sharp FA, et al. Autophagy controls IL-1beta secretion by targeting pro-IL-1beta for degradation. J Biol Chem (2011) 286:9587-97. doi:10.1074/jbc.M110.202911

52. Shi C-S, Shenderov K, Huang N-N, Kabat J, Abu-Asab M, Fitzgerald KA, et al. Activation of autophagy by inflammatory signals limits IL-1beta production by targeting ubiquitinated inflammasomes for destruction. Nat Immunol (2012) 13:255-63. doi:10.1038/ni.2215

53. Virgin HW, Levine B. Autophagy genes in immunity. Nat Immunol (2009) 10:461-70. doi:10.1038/ni.1726

54. Liu F, Lee JY, Wei H, Tanabe O, Engel JD, Morrison SJ, et al. FIP200 is required for the cell-autonomous maintenance of fetal hematopoietic stem cells. Blood (2010) 116:4806-14. doi:10.1182/blood-2010-06-288589

55. Pua HH, Dzhagalov I, Chuck M, Mizushima N, He YW. A critical role for the autophagy gene Atg5 in T cell survival and proliferation. J Exp Med (2007) 204:25-31. doi:10.1084/jem.20061303

56. Bell BD, Leverrier S, Weist BM, Newton RH, Arechiga AF, Luhrs KA, et al. FADD and caspase- 8 control the outcome of autophagic signaling in proliferating T cells. Proc Natl Acad Sci U S A (2008) 105:16677-82. doi:10.1073/pnas. 0808597105

57. McLeod IX, Jia W, He Y-W. The contribution of autophagy to lymphocyte survival and homeostasis. Immunol Rev (2012) 249:195-204. doi:10.1111/j.1600065X.2012.01143.x

58. Willinger T, Flavell RA. Canonical autophagy dependent on the class III phosphoinositide- 3 kinase Vps34 is required for naive T-cell homeostasis. Proc Natl Acad Sci U S A (2012) 109:8670-5. doi:10.1073/pnas.1205305109

59. Parekh VV, Wu L, Boyd KL, Williams JA, Gaddy JA, Olivares-Villagómez D, et al. Impaired autophagy, defective $\mathrm{T}$ cell homeostasis, and a wasting syndrome in mice with a T cell-specific deletion of Vps34. J Immunol (2013) 190:5086-101. doi:10.4049/jimmunol.1202071

60. Hubbard VM, Valdor R, Patel B, Singh R, Cuervo AM, Macian F. Macroautophagy regulates energy metabolism during effector T cell activation. J Immunol (2010) 185:7349-57. doi:10.4049/jimmunol.1000576

61. Miller BC, Zhao Z, Stephenson LM, Cadwell K, Pua HH, Lee HK, et al. The autophagy gene ATG5 plays an essential role in B lymphocyte development. Autophagy (2008) 4:309-14.

62. Paludan C, Schmid D, Landthaler M, Vockerodt M, Kube D, Tuschl T, et al. Endogenous MHC class II processing of a viral nuclear antigen after autophagy. Science (2005) 307:593-6. doi:10.1126/science.1104904

63. Schmid D, Pypaert M, Münz C. Antigen-loading compartments for major histocompatibility complex class II molecules continuously receive input from autophagosomes. Immunity (2007) 26:79-92. doi:10.1016/j.immuni.2006.10. 018

64. Jagannath C, Lindsey DR, Dhandayuthapani S, Xu Y, Hunter RL Jr, Eissa NT. Autophagy enhances the efficacy of BCG vaccine by increasing peptide presentation in mouse dendritic cells. Nat Med (2009) 15:267-76. doi:10.1038/nm.1928

65. Lee HK, Mattei LM, Steinberg BE, Alberts P, Lee YH, Chervonsky A, et al. In vivo requirement for Atg 5 in antigen presentation by dendritic cells. Immunity (2010) 32:227-39. doi:10.1016/j.immuni.2009.12.006

66. Nedjic J, Aichinger M, Emmerich J, Mizushima N, Klein L. Autophagy in thymic epithelium shapes the T-cell repertoire and is essential for tolerance. Nature (2008) 455:396-400. doi:10.1038/nature07208

67. Li Y, Wang LX, Yang G, Hao F, Urba WJ, Hu HM. Efficient cross-presentation depends on autophagy in tumor cells. Cancer Res (2008) 68:6889-95. doi:10. 1158/0008-5472.CAN-08-0161

68. Crotzer VL, Blum JS. Autophagy and adaptive immunity. Immunology (2010) 131:9-17. doi:10.1111/j.1365-2567.2010.03321.x

69. Pandey UB, Nie Z, Batlevi Y, McCray BA, Ritson GP, Nedelsky NB, et al. HDAC6 rescues neurodegeneration and provides an essential link between autophagy and the UPS. Nature (2007) 447:859-63. doi:10.1038/nature05853

70. Conway KL, Kuballa P, Khor B, Zhang M, Shi HN, Virgin HW, et al. ATG5 regulates plasma cell differentiation. Autophagy (2013) 9(4):528-37. doi:10.4161/ auto. 23484
71. Cortes M, Georgopoulos K. Aiolos is required for the generation of high affinity bone marrow plasma cells responsible for long-term immunity. J Exp Med (2004) 199:209-19. doi:10.1084/jem.20031571

72. Shapiro-Shelef M, Lin K-I, Savitsky D, Liao J, Calame K. Blimp-1 is required for maintenance of long-lived plasma cells in the bone marrow. J Exp Med (2005) 202:1471-6. doi:10.1084/jem.20051611

73. Hu C-CA, Dougan SK, McGehee AM, Love JC, Ploegh HL. XBP-1 regulates signal transduction, transcription factors and bone marrow colonization in B cells. EMBO J (2009) 28:1624-36. doi:10.1038/emboj.2009.117

74. Peperzak V, Vikstrom I, Walker J, Glaser SP, LePage M, Coquery CM, et al. Mcl1 is essential for the survival of plasma cells. Nat Immunol (2013) 14:290-7. doi:10.1038/ni.2527

75. Pengo N, Cenci S. The role of autophagy in plasma cell ontogenesis. Autophagy (2013) 9:942-4. doi:10.4161/auto.24399

76. Sprocati T, Ronchi P, Raimondi A, Francolini M, Borgese N. Dynamic and reversible restructuring of the ER induced by PDMP in cultured cells. J Cell Sci (2006) 119:3249-60. doi:10.1242/jcs.03058

77. Fujita E, Kouroku Y, Isoai A, Kumagai H, Misutani A, Matsuda C, et al. Two endoplasmic reticulum-associated degradation (ERAD) systems for the novel variant of the mutant dysferlin: ubiquitin/proteasome $\operatorname{ERAD}(\mathrm{I})$ and autophagy/lysosome ERAD(II). Hum Mol Genet (2007) 16:618-29. doi:10.1093/ hmg/ddm002

78. Ishida Y, Yamamoto A, Kitamura A, Lamande SR, Yoshimori T, Bateman JF, et al. Autophagic elimination of misfolded procollagen aggregates in the endoplasmic reticulum as a means of cell protection. Mol Biol Cell (2009) 20:2744-54 doi:10.1091/mbc.E08-11-1092

79. Hsu MC, Toellner KM, Vinuesa CG, MacLennan IC. B cell clones that sustain long-term plasmablast growth in T-independent extrafollicular antibody responses. Proc Natl Acad Sci U S A (2006) 103:5905-10. doi:10.1073/pnas. 0601502103

80. Levine B, Kroemer G. Autophagy in the pathogenesis of disease. Cell (2008) 132:27-42. doi:10.1016/j.cell.2007.12.018

81. Mathew R, Kongara S, Beaudoin B, Karp CM, Bray K, Degenhardt K, et al. Autophagy suppresses tumor progression by limiting chromosomal instability. Genes Dev (2007) 21:1367-81. doi:10.1101/gad.1545107

82. Karantza-Wadsworth V, Patel S, Kravchuk O, Chen G, Mathew R, Jin S, et al. Autophagy mitigates metabolic stress and genome damage in mammary tumorigenesis. Genes Dev (2007) 21:1621-35. doi:10.1101/gad.1565707

83. Maiuri MC, Tasdemir E, Criollo A, Morselli E, Vicencio JM, Carnuccio R, et al. Control of autophagy by oncogenes and tumor suppressor genes. Cell Death Differ (2009) 16:87-93. doi:10.1038/cdd.2008.131

84. Degenhardt K, Mathew R, Beaudoin B, Bray K, Anderson D, Chen G, et al. Autophagy promotes tumor cell survival and restricts necrosis, inflammation and tumorigenesis. Cancer Cell (2006) 10:51-64. doi:10.1016/j.ccr.2006.06.001

85. Maiuri MC, Zalckvar E, Kimchi A, Kroemer G. Self-eating and self-killing: crosstalk between autophagy and apoptosis. Nat Rev Mol Cell Biol (2007) 8:741-52. doi:10.1038/nrm 2239

86. Goldberg AL. Development of proteasome inhibitors as research tools and cancer drugs. J Cell Biol (2012) 199(4):583-8. doi:10.1083/jcb.201210077

87. Hideshima T, Anderson KC. Biologic impact of proteasome inhibition in multiple myeloma cells-from the aspects of preclinical studies. Semin Hematol (2012) 49(3):223-7. doi:10.1053/j.seminhematol.2012.04.006

88. Aronson LI, Davies FE. DangER: protein ovERload. Targeting protein degradation to treat myeloma. Haematologica (2012) 97:1119-30. doi:10.3324/ haematol.2012.064923

89. Bianchi G, Oliva L, Cascio P, Pengo N, Fontana F, Cerruti F, et al. The proteasome load versus capacity balance determines apoptotic sensitivity of multiple myeloma cells to proteasome inhibition. Blood (2009) 113:3040-9. doi:10.1182/blood-2008-08-172734

90. Yewdell JW, David A. Proteostenosis: cancer's Achilles heel? J Leukoc Biol (2012) 92:913-5. doi:10.1189/jlb.0612272

91. Leleu X, Xu L, Jia X, Sacco A, Farag M, Hunter ZR, et al. Endoplasmic reticulum stress is a target for therapy in Waldenstrom macroglobulinemia. Blood (2009) 113:626-34. doi:10.1182/blood-2007-10-116848

92. Leleu X, Eeckhoute J, Jia X, Roccaro AM, Moreau AS, Farag M, et al. Targeting NF-kappaB in Waldenstrom macroglobulinemia. Blood (2008) 111:5068-77. doi:10.1182/blood-2007-09-115170 
93. Roccaro AM, Sacco A, Aujay M, Ngo HT, Azab AK, Azab F, et al. Selective inhibition of chymotrypsin-like activity of the immunoproteasome and constitutive proteasome in Waldenstrom macroglobulinemia. Blood (2010) 115:4051-60. doi:10.1182/blood-2009-09-243402

94. Ghobrial IM, Xie W, Padmanabhan S, Badros A, Rourke M, Leduc R, et al. Phase II trial of weekly bortezomib in combination with rituximab in untreated patients with Waldenström macroglobulinemia. Am J Hematol (2010) 85:670-4. doi:10.1002/ajh.21788

95. Dimopoulos MA, García-Sanz R, Gavriatopoulou M, Morel P, Kyrtsonis MC, Michalis E, et al. Primary therapy of Waldenstrom macroglobulinemia (WM) with weekly bortezomib, low-dose dexamethasone, and rituximab (BDR): longterm results of a phase 2 study of the European Myeloma Network (EMN). Blood (2013) 122:3276-82. doi:10.1182/blood-2013-05-503862

96. Hoang B, Benavides A, Shi Y, Frost P, Lichtenstein A. Effect of autophagy on multiple myeloma cell viability. Mol Cancer Ther (2009) 8:1974-84. doi:10.1158/ 1535-7163.MCT-08-1177

97. Kawaguchi T, Miyazawa K, Moriya S, Ohtomo T, Che X-F, Naito M, et al. Combined treatment with bortezomib plus bafilomycin Al enhances the cytocidal effect and induces endoplasmic reticulum stress in U266 myeloma cells: crosstalk among proteasome, autophagy-lysosome and ER stress. Int J Oncol (2011) 38:643-54. doi:10.3892/ijo.2010.882

98. Santo L, Hideshima T, Kung AL, Tseng J-C, Tamang D, Yang M, et al. Preclinical activity, pharmacodynamic, and pharmacokinetic properties of a selective
HDAC6 inhibitor, ACY-1215, in combination with bortezomib in multiple myeloma. Blood (2012) 119:2579-89. doi:10.1182/blood-2011-10-387365

99. Lamy L, Ngo VN, Emre NCT, Shaffer AL III, Yang Y, Tian E, et al. Control of autophagic cell death by caspase-10 in multiple myeloma. Cancer Cell (2013) 23:435-49. doi:10.1016/j.ccr.2013.02.017

Conflict of Interest Statement: The authors declare that the research was conducted in the absence of any commercial or financial relationships that could be construed as a potential conflict of interest.

Received: 13 January 2014; accepted: 26 February 2014; published online: 12 March 2014.

Citation: Oliva L and Cenci S (2014) Autophagy in plasma cell pathophysiology. Front. Immunol. 5:103. doi: 10.3389/fimmu.2014.00103

This article was submitted to B Cell Biology, a section of the journal Frontiers in Immunology.

Copyright (c) 2014 Oliva and Cenci. This is an open-access article distributed under the terms of the Creative Commons Attribution License (CC BY). The use, distribution or reproduction in other forums is permitted, provided the original author(s) or licensor are credited and that the original publication in this journal is cited, in accordance with accepted academic practice. No use, distribution or reproduction is permitted which does not comply with these terms. 